\title{
Quick-Freeze/Deep-Etch Visualization of Age-Related Lipid Accumulation in Bruch's Membrane
}

\author{
Jeffrey W. Ruberti, ${ }^{1}$ Christine A. Curcio, ${ }^{2}$ C. Leigh Millican, ${ }^{3}$ Bert P. M. Menco, ${ }^{4}$ \\ Jiabn-Dar Huang, ${ }^{1}$ and Mark Jobnson ${ }^{1}$
}

Purpose. To examine age-related changes in the ultrastructure of Bruch's membrane with quick-freeze/deep-etch (QFDE) and conventional thin-section transmission electron microscopy (TEM).

Methods. Four eyes from human donors aged 27, 41, 76, and 78 years were preserved within 4 hours of death. Full-thickness tissue blocks from the macula were prepared for TEM or for QFDE.

RESULTs. Ultrastructure seen by conventional TEM was revealed in greater detail by QFDE. Cholesterol-containing particles (mean diameter, $80 \mathrm{~nm}$ ) formed a thin densely packed layer external to the basal lamina of the retinal pigment epithelium (RPE) only in older eyes. The mesh size of the RPE basal lamina was smaller than the particles, and it appeared larger in older eyes. QFDE also revealed less decorated collagen fibrils in older eyes.

Conclusions. The data suggest that the predilection of a extremely thin sublayer of inner Bruch's membrane for accumulating lipid particles may eventually lead to a confluent lipid wall capable of isolating the retina from its blood supply. If these lipids originate in the retinal pigment epithelium, then they are unlikely to have passed through the basal lamina in this form. The age-related increase in lipid particles corresponds with an age-related increase in hydraulic resistance determined in excised Bruch's membrane/choroid by others. QFDE will be useful for future modeling studies of Bruch's membrane transport and to identify those moieties responsible for deleterious age-related transport changes in Bruch's membrane. (Invest Opbthalmol Vis Sci. 2003;44:1753-1759) DOI:10.1167/iovs.02-0496

ge-related maculopathy (ARM) is currently the leading
cause of new loss of vision among older adults and will
become a major public health concern as the population of the
industrialized world ages. ${ }^{1}$ The most prominent clinical and
histopathologic lesions of ARM involve Bruch's membrane, a
thin specialized vascular intima between the retinal pigment

From the Departments of ${ }^{1}$ Biomedical Engineering and ${ }^{4}$ Neurobiology and Physiology, Northwestern University, Evanston, Illinois; the ${ }^{2}$ Department of Ophthalmology, University of Alabama School of Medicine, Birmingham, Alabama; and the ${ }^{3}$ High Resolution Imaging Facility, University of Alabama at Birmingham, Birmingham, Alabama.

Supported by internal funds at Northwestern University, National Eye Institute Grant EY06109, National Science Foundation IBN0094709, and a grant from Macular Degeneration Research Program of the American Health Assistance Foundation. CAC holds a Lew R.Wasserman Merit award from Research to Prevent Blindness, Inc.

Submitted for publication May 24, 2002; revised November 12, 2002; accepted December 6, 2002.

Commercial relationships policy: $\mathrm{N}$.

The publication costs of this article were defrayed in part by page charge payment. This article must therefore be marked "advertisement" in accordance with 18 U.S.C. $\$ 1734$ solely to indicate this fact.

Corresponding author: Mark Johnson, Department of Biomedical Engineering, Northwestern University, 2145 Sheridan Road, Evanston, IL 60208; m-johnson2@northwestern.edu. epithelium (RPE) and the choriocapillaris, the blood supply of the photoreceptors. The only established risk factor for early ARM is advanced age, which brings prominent changes to the macular region of this membrane. These include a threefold thickening, decreased collagen solubility and metalloproteinase activity, and accumulation of debris, advanced glycation end products, and extracellular lipids, including esterified and unesterified cholesterol. ${ }^{2-15}$

Unlike age-related accumulation of cholesterol in other connective tissues (cornea, sclera, and arterial intima), ${ }^{16-20}$ that which occurs in Bruch's membrane is apparently unique in its potential impact on fluid and nutrient exchange. According to the lipid-barrier hypothesis, this accumulation renders Bruch's membrane increasingly hydrophobic, impeding translocation of hydrophilic substances to and from the choriocapillaris. ${ }^{7,21,22}$ Impaired movement of fluids, either derived from RPE or from choroidal neovascularization, may contribute to the formation of RPE detachments in patients with ARM. ${ }^{22}$ It may also prevent adequate replenishment of photoreceptors with plasma-derived retinoid derivatives, thus contributing to age- and ARM-related photoreceptor dysfunction. ${ }^{23}$

The lipid-barrier hypothesis would be strengthened by a morphologic demonstration of this barrier in human Bruch's membrane. Empirical studies ${ }^{24,25}$ suggest a two-phase decline in the hydraulic conductivity of isolated Bruch's membrane with a marked decline between birth and 40 years of age and a slower decline after that age. However, the structural basis for this decline has not been established. The decline in hydraulic conductivity in younger eyes has been attributed to increased collagen cross-linking, ${ }^{24}$ but no ultrastructural evidence supporting this idea is yet available. Throughout adulthood, Bruch's membrane accumulates solid particles containing esterified and unesterified cholesterol, roughly $100 \mathrm{~nm}$ in diameter, that form a discrete sublayer external to the RPE basal lamina in eyes of many persons older than 60 years. ${ }^{2}$

Determining how esterified cholesterol-containing particles interact with each other and extracellular matrix components to form a hydrophobic barrier and reconciling the different time courses of changes in the hydraulic conductivity and lipid content of Bruch's membrane would be facilitated by a method that preserves ultrastructure of lipids and extracellular matrix. The ultrastructure of Bruch's membrane has been examined extensively by conventional thin-section transmission electron microscopy (TEM), but the dehydration and embedding steps of tissue processing optimally preserve neither the small lipid particles nor the ultrastructure of the extracellular matrix. ${ }^{26,27}$ Bruch's membrane has also been examined using the osmiumtannic acid-paraphenylenediamine method to preserve lipid particles, but this method does not improve visualization of extracellular matrix components. ${ }^{2}$

Therefore, we examined macular Bruch's membrane of normal human eyes by quick-freeze/deep-etch (QFDE) microscopy, a method that provides a quasi-three-dimensional view of connective tissue ultrastructure and lipid particles in exquisite detail. 


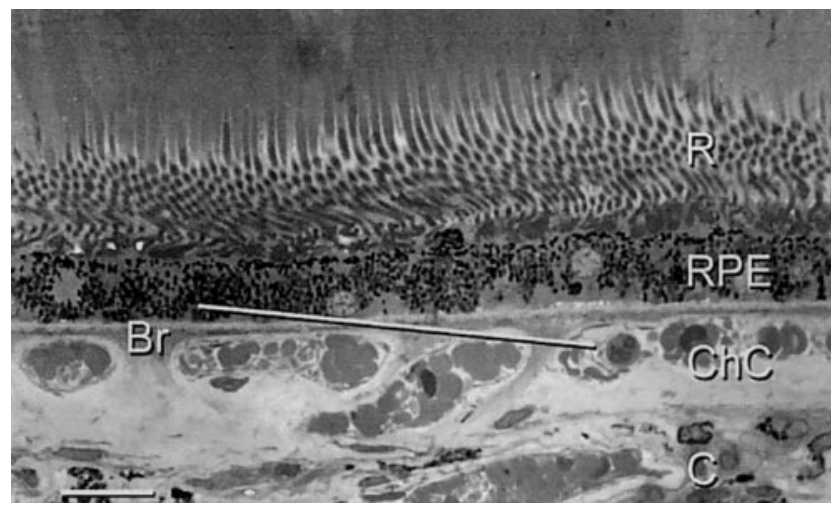

FiguRE 1. Light micrograph of a human retina. The line shows the approximate plane of fracture for QFDE and oblique sections for thin-section TEM. In actual samples the retina was removed from the RPE before ultrarapid freezing. Specimens were sectioned at $1 \mu \mathrm{m}$ and stained with $2 \%$ toluidine blue in $2 \%$ sodium borate. R, retina; RPE, retinal pigment epithelium; $\mathrm{Br}$, Bruch's membrane; $\mathrm{ChC}$, choriocapillaris; C, choroid. Bar, $20 \mu \mathrm{m}$.

\section{Methods}

We used both thin-section TEM and QFDE to examine RPE and Bruch's membrane from eyes of normal human donors. Four eyes of donors aged $27,41,76$, and 78 years were preserved within 4 hours of death. Anterior segments were removed from the eyes, and posterior segments were fixed by immersion for at least 24 hours at $4^{\circ} \mathrm{C}$ in $0.1 \mathrm{M}$ phosphate buffer ( $\mathrm{pH} 7.4$ ) with $2.5 \%$ glutaraldehyde and $1 \%$ paraformaldehyde.

Maculas, including retina, choroid, and sclera, were dissected into $2 \times 2$ - $\mathrm{mm}^{2}$ blocks and stored in $0.1 \mathrm{M}$ phosphate buffer. One block adjacent to the fovea from each eye was prepared for light microscopic and conventional thin-section TEM examination. These tissue blocks were postfixed for 1 hour at $25^{\circ} \mathrm{C}$ in $1 \%$ osmium and $0.125 \%$ potassium ferricyanide, dehydrated through ethanol and propylene oxide, and embedded in resin (PolyBed 812; Polysciences, Warrington, PA). Blocks were then sectioned at $1 \mu \mathrm{m}$ in the vertical or oblique planes and stained with $1 \%$ toluidine blue and sodium borohydrate. The $1-\mu \mathrm{m}$ sections were evaluated for disease. Thin sections from each tissue block were cut and poststained with uranyl acetate and lead citrate for thin-section TEM examination.

The other macular tissue blocks were processed for QFDE. For each specimen, the retina was lifted from the underlying RPE. The specimen was placed on an aluminum tab and slam frozen (Gentleman Jim Slam Freezing Apparatus ${ }^{28,29}$ ) RPE side down, onto a copper block cooled to liquid nitrogen temperature $\left(-195^{\circ} \mathrm{C}\right)$. The frozen specimen was then stored in liquid nitrogen until further use.

After transfer into a freeze-fracture apparatus (model CFE-40; Cressington Scientific Instruments, Watford, UK), the specimen was fractured obliquely through RPE cells, etched for 25 minutes at $-95^{\circ} \mathrm{C}$, and rotary shadowed with a platinum-carbon mixture at a $20^{\circ}$ angle and then strengthened with carbon evaporated from a $90^{\circ}$ angle overhead. In accordance with the method of Ou et al.,$^{30}$ the replicated specimen was coated with collodion in $2 \%$ amyl acetate and placed in household bleach for digestion overnight. The bleach-cleaned replica was picked up on either nickel or copper mesh hexagonal grids and washed in amyl acetate to remove the collodion.

Thin sections and replicas were viewed on two transmission electron microscopes (model 1200 EX II and JEM-100 CX TEMSCAN microscopes, respectively; JEOL, Tokyo, Japan). An oblique fracture plane passing nearly parallel to the retina, as shown in Figure 1, gave the best view of Bruch's membrane ultrastructure.

Morphometric measurements were performed on QFDE images that were scanned into a TIFF format with a flatbed image scanner (model SilverScan II; LaCie US, Hillsboro, OR). Image analysis was performed using NIH Image (available by ftp from zippy.nimh.nih. gov/or from http://rsb.info.nih.gov/nih-image; developed by Wayne Rasband, National Institutes of Health, Bethesda, MD). Particle diameters were determined as the geometric mean of the major and minor axis of the lipid particle. The number of lipid-rich particles in the basal lamina per micrograph was graded for 16 micrographs by one observer, as follows: 0 , none; + , low; ++ , moderate; and +++ , high. Micrographs were not masked, because the micrographs of older eyes were easily distinguished from those of young eyes.

\section{Results}

Before preparation for conventional thin-section TEM and QFDE, the globes were inspected internally with epi- and retroillumination, and the maculas were determined to lack grossly visible drusen or pigmentary change. However, it was found on histopathologic evaluation that the two older eyes had small patches of basal laminar deposit located between the RPE and its basal lamina, a typical finding for eyes of this age. ${ }^{10}$ The eye of the 76-year-old donor also had numerous small protuberances on the inner surface of Bruch's membrane that were too small to be considered drusen.

Oblique sections of Bruch's membrane, as viewed by TEM, are shown for a 27-year-old eye (Fig. 2A), a 41-year-old eye (Fig. 3A), a 76-year-old eye (Fig. 3C), and a 78-year-old eye (Fig. 2C). In the younger eyes, the basal infoldings of the RPE, the RPE basal lamina and the inner collagenous layer (ICL) are apparent (Figs. 2A, 3A). Collagen fibrils and an unidentified banded material were apparent within the ICL. The ends of collagen fibrils could be seen closely apposed to, perhaps embedded in, the RPE basal lamina (Fig. 3A). Consistent with previous TEM descriptions, the most prominent differences between the older (Figs. 2C, 3C) and younger (Figs. 2A, 3A) eyes were disorganization or disappearance of the RPE basal infoldings, increased electron density of the ICL, a paucity of collagen fibrils immediately adjacent to the RPE basal lamina, and the presence of numerous round electron-lucent profiles. These profiles were distributed along fibrils in the ICL and in a band between the ICL and the RPE basal lamina in the older donors (Figs. 2C, 3C). This band, two to four particles thick in crosssections of many older eyes, ${ }^{2}$ looks wide in Figures $2 \mathrm{C}$ and $3 \mathrm{C}$, because of the oblique plane of section. These profiles were previously identified as cholesterol-containing particles, because they are solid when viewed with lipid-preserving ultrastructural techniques, they are extractable with lipid solvents, and they become more numerous with age along with histochemically detectable and directly assayed esterified and unesterified cholesterol. ${ }^{2}$

Oblique fractures of Bruch's membrane viewed in QFDE preparations of a 27-year-old eye (Fig. 2B), a 41-year-old eye (Fig. 3B), a 76-year-old eye (Fig. 3D), and a 78-year-old eye (Fig. 2D) revealed the same ultrastructural features, but in much greater detail. The RPE basal lamina appeared as a dense meshwork, and the ICL contained a loose network of 45-nm diameter fibrils. Of note were spherical and elliptical particles $80.8 \pm 20.7 \mathrm{~nm}$ (mean $\pm \mathrm{SD}$ ) in diameter that were minimally etched by the QFDE process and found in abundance only in the older eyes (Figs. 2D, 3D). In all the micrographs of the older eyes, low density $(+)$ of these particles was found in the basal lamina itself and high density $(+++)$ was found in the region between the basal lamina and the ICL. Because these particles were solid, of similar dimensions, and present in the same locations and in the same age group as those seen in thin-section TEM, we identified them as EC-containing particles.

More lipid particles appear embedded in the basal lamina in the thin-section TEM images (Figs. 2C, 3C) than in the QFDE 

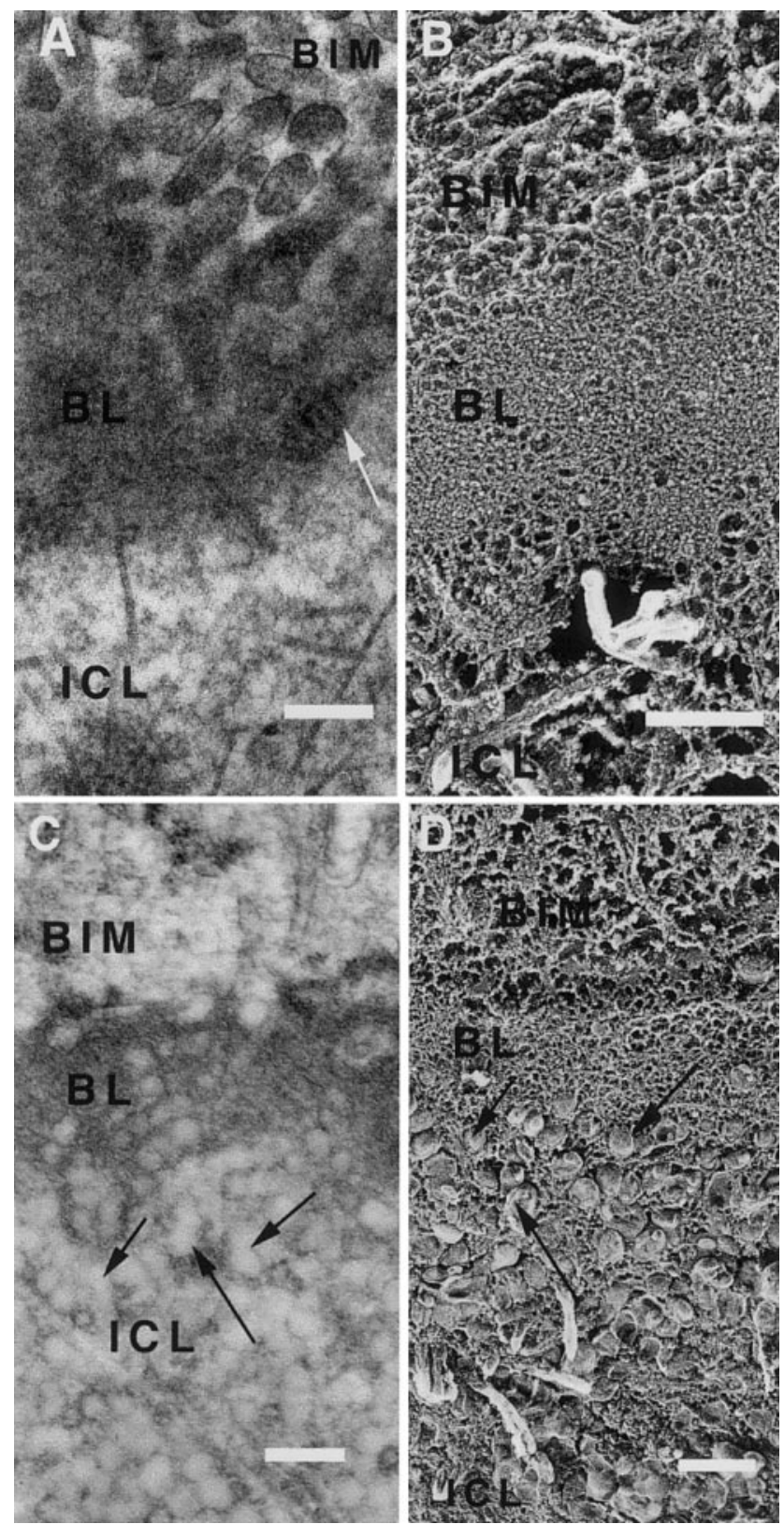

Figure 2. Oblique standard thin-section TEM $(\mathbf{A}, \mathbf{C})$ and QFDE $(\mathbf{B}, \mathbf{D})$ micrographs of the RPE/Bruch's membrane interface from a young (27-year-old: A, B) and an older (78-year-old: C, D) eye. Basal lamina (BL) is between the ICL and the basal infolding membrane (BIM) of the RPE cells. In the younger eye, banded material was present in the basal lamina (white arrow). In the older eye, lipid rich particles (black arrows) were present at the choriocapillaris (external) side of the RPE basal lamina. The electron-lucent particles in thin-section TEM micrographs roughly correspond to solid particles in QFDE images. Scale bars, $300 \mathrm{~nm}$.

images (Figs. 2D, 3D). The apparent discrepancy between the two imaging modalities can be reconciled by considering Figure $2 \mathrm{~A}$, in which RPE basal infoldings also appear to be embedded in the basal lamina. This underscores that sections imaged by thin-section TEM are sufficiently thick to permit the superimposition of two very thin sublayers. Therefore it is likely that Figures 2C and 3C represent superimposition of the RPE basal lamina and the adjacent layer of Bruch's membrane rather than numerous particles embedded in basal lamina.
The main difference between the two older eyes examined was the number of these lipid particles. At the interface between the basal lamina and the ICL of the 76-year-old donor eye was a wall of tightly packed lipid particles appreciable by both thin-section TEM (Fig. 3C) and QFDE (Fig. 3D). This wall was

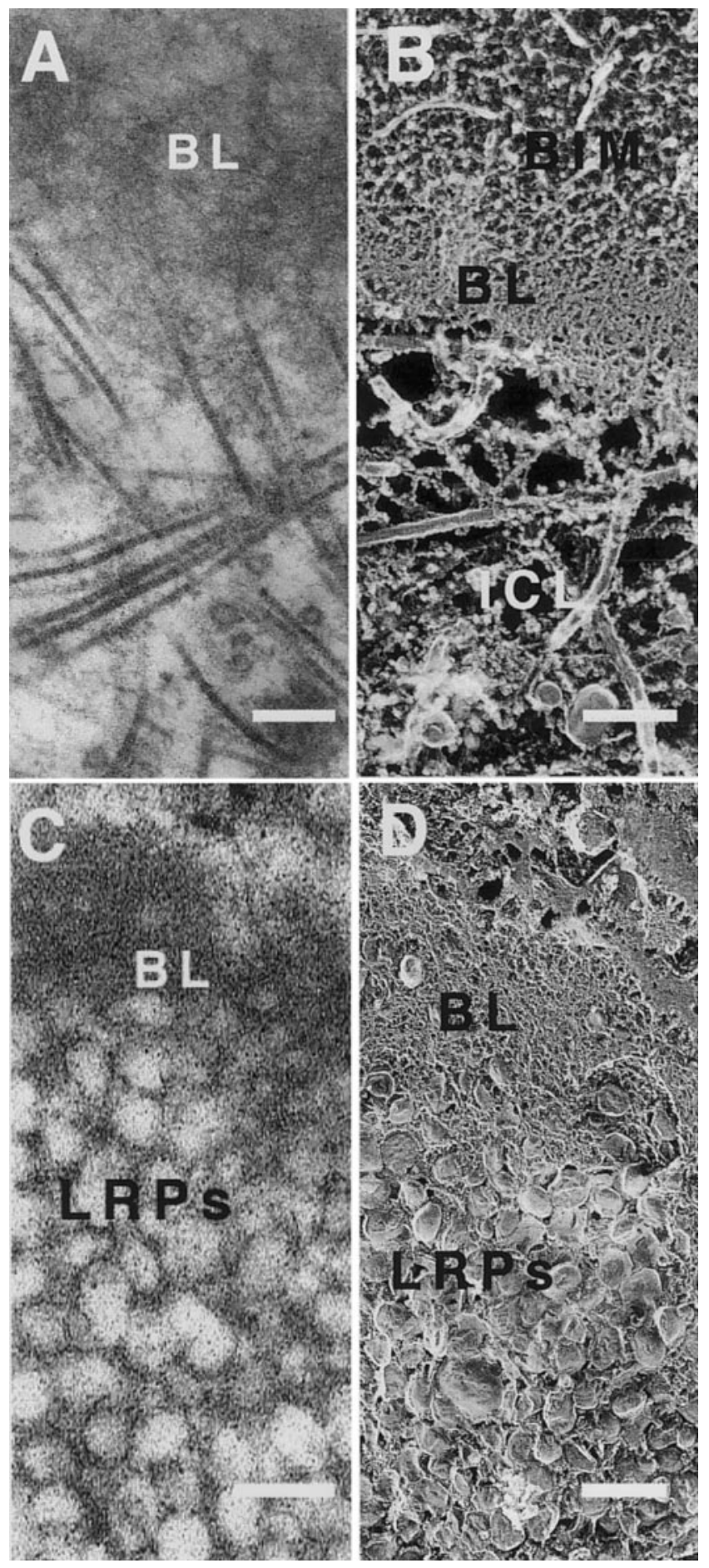

FIGURE 3. Oblique standard thin-section TEM $(\mathbf{A}, \mathbf{C})$ and QFDE $(\mathbf{B}, \mathbf{D})$ micrographs of the RPE-Bruch's membrane interface from a young (41-year-old: A, B) and an older (76-year-old: C, D) eye. Labels as in Figure 2 . Although the ICL was relatively open in the younger eye a tightly packed layer of lipid-rich particles (black arrows) was evident on the choriocapillaris side of the RPE basal lamina in the older eye. The electron-lucent particles in thin-section TEM images roughly correspond to solid particles in QFDE images. Scale bars, $300 \mathrm{~nm}$. 


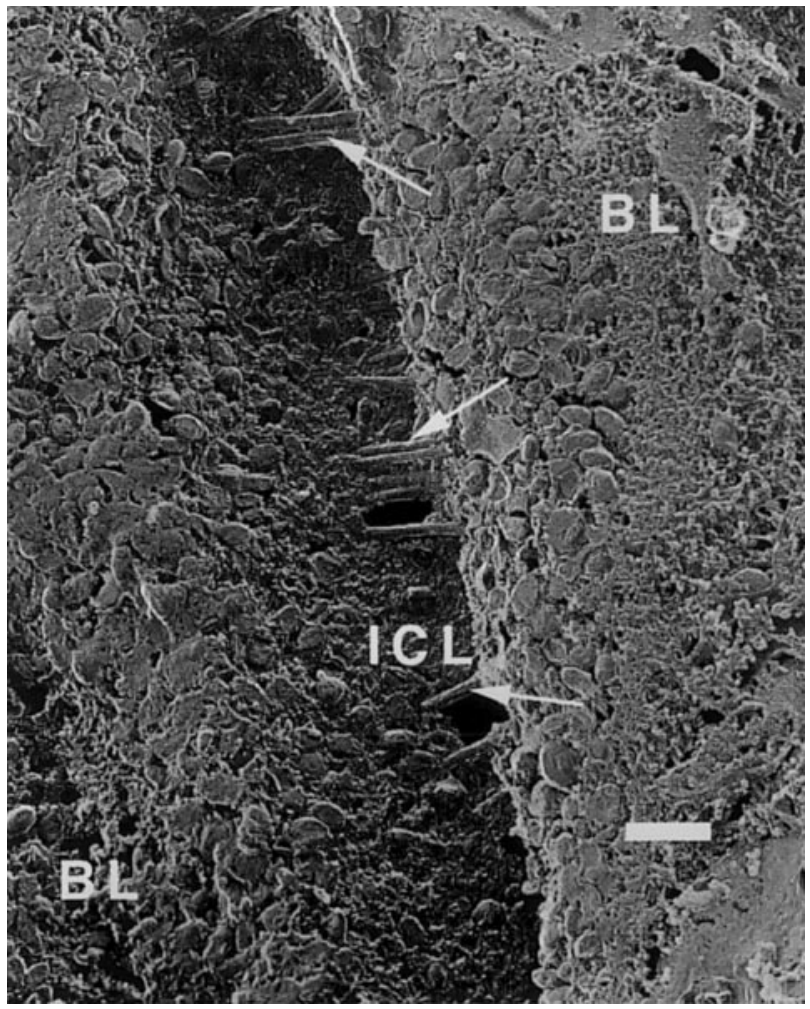

FigURE 4. A wall of lipid-rich particles embedded in the matrix just below the RPE basal lamina (BL) in a 76-year-old eye. In the center of the micrograph, $50 \mathrm{~nm}$ collagen fibrils (arrows) of the ICL of Bruch's are visible. Scale bar, $300 \mathrm{~nm}$. not apparent in the 78-year-old eye (Figs. 2C, 2D). Figure 4 demonstrates the high density of these embedded lipid rich particles showing a region where the fracture plane jumped from the basal lamina down to the ICL (the bottom of the groove) and then back up to the basal lamina.

In addition to extending results from a previous study using sections viewed by conventional thin-section and lipid-preserving TEM, ${ }^{2}$ QFDE also revealed new details about the morphology of both the lipid-rich particles and the extracellular matrix containing them. An exterior shell was apparent on many lipid-rich particles (Fig. 5). Regarding the extracellular matrix, QFDE revealed a dense weave (interfibril spacing $<15 \mathrm{~nm}$ ) in the RPE basal lamina of the younger eyes and a perhaps somewhat less dense weave in the older eyes (Figs. 6, 7). A further age-related difference involved the surface of collagen fibrils. These fibrils displayed an extensive fine-scale decoration ${ }^{31}$ in the younger eyes (Fig. 8A) that was reduced or absent in the older eyes (Fig. 8B). Collagen was identified by its characteristic banding pattern. Elastin-like material identification was based on correspondence with standard thin-section TEM images of the same regions.

\section{Discussion}

Our results demonstrate that the QFDE method provides highresolution images of age-related changes in the Bruch's membrane ultrastructure. Many of the changes revealed by QFDE are directly corroborated by standard thin-section TEM images of the same maculas and by numerous thin-section TEM images published in the literature..$^{2,11-13,32}$ QFDE minimizes the loss of the extracellular matrix and lipids that often accompanies dehydration through graded alcohol, ${ }^{26,27}$ providing a clearer view of these structures than traditional methods of preparation for TEM. Because only four eyes were examined in this study, results found only in the QFDE images should be interpreted cautiously. Nevertheless, the QFDE images demonstrate the potential power of this method for detailed analysis of

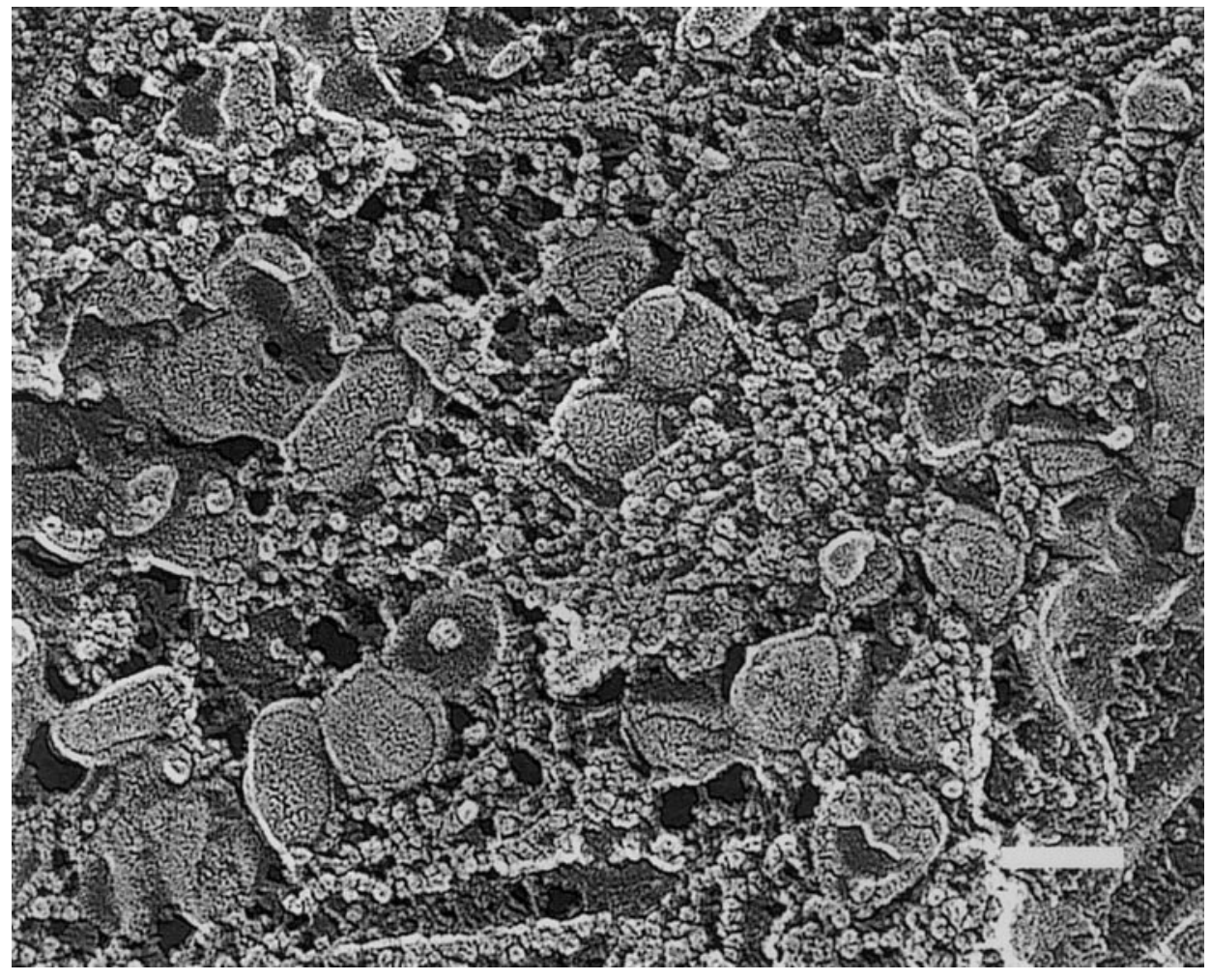

FIGURE 5. QFDE image of lipids in a region adjacent to RPE basal lamina of 78-year-old eye showing ultrastructural detail of lipid- extracellular matrix interaction. The lipid rich particles $(\sim 80 \mathrm{~nm}$ in diameter) appear intimately involved with matrix molecules. Scale bar, $100 \mathrm{~nm}$. 

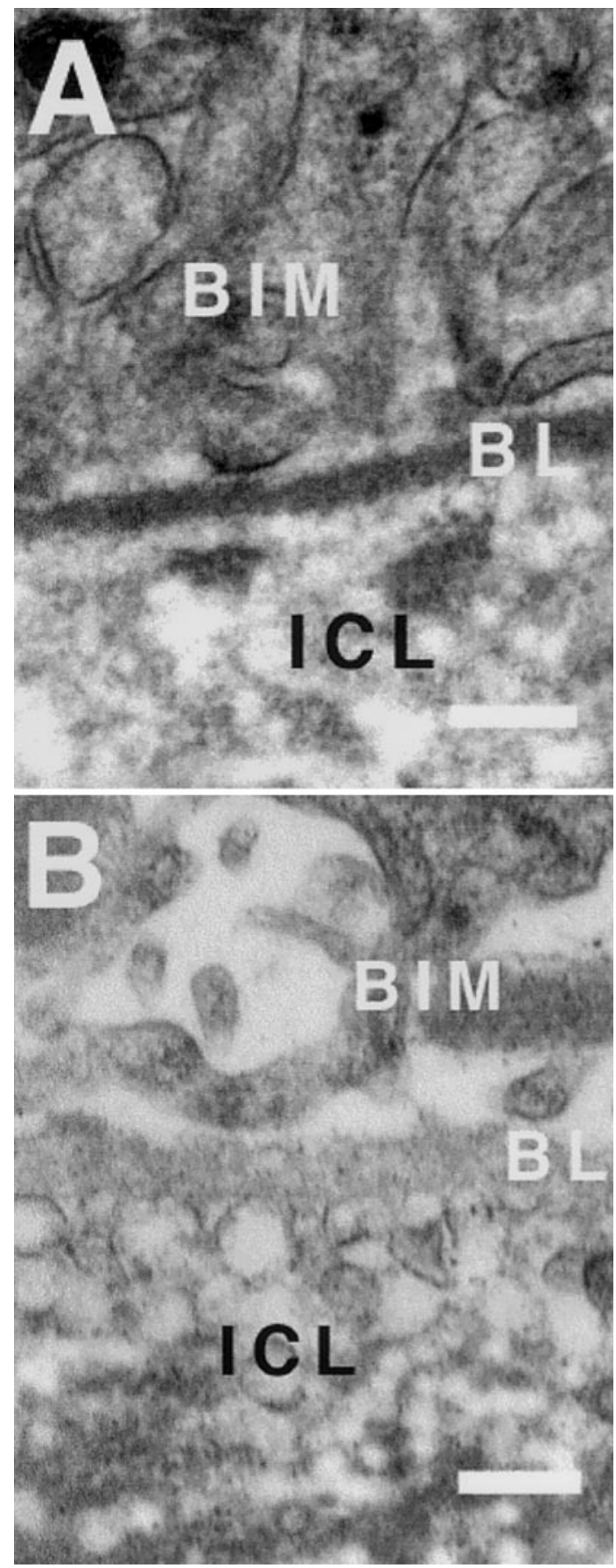

FIGURE 6. Standard thin-section TEM cross-sections through a (A) 27-year-old and (B) 78-year-old human RPE basal lamina (BL). Note the compactness and dense staining of young RPE basal lamina. In the older eye, the basal lamina appeared diffuse with indistinct borders. $\mathrm{BIM}$, basal infolding membrane of RPE cells. Scale bar, $300 \mathrm{~nm}$.

ultrastructural changes in Bruch's membrane due to aging and disease.

Our most prominent finding is the markedly improved visualization of cholesterol-containing particles accumulated in a discrete sublayer external to the RPE basal lamina, a region we call the lipid wall. Previous work has shown an age-related increase of esterified cholesterol by filipin histochemistry and the presence of solid lipid particles by a lipid-preserving ultrastructural method in this region. ${ }^{2}$ Notably, the present study showed the packing of lipid particles to be so dense that only a small fraction of the volume of this layer appeared to be available for transport. Marshall et al. ${ }^{22}$ determined that the hydraulic resistance (the inverse of the hydraulic conductivity) of Bruch's membrane was closely correlated to lipid accumulation. Our data support the possibility that the age-related increase of the hydraulic resistance of Bruch' $s$ membrane ${ }^{22}$ is closely related to formation of the lipid wall. A detailed transport analysis has yet to be conducted, but transport of water and hydrophilic moieties across Bruch's membrane would be expected to be considerably compromised by the barrier shown in Figures 3C, 3D, and 4.

In this investigation, QFDE revealed previously unappreciated details of lipid particle substructure, showing both exterior and interior components (Fig. 5). Lipid particles with external shells were also seen in QFDE studies of cholesterol accumulation in the aortic intima of rabbit after a bolus injec-

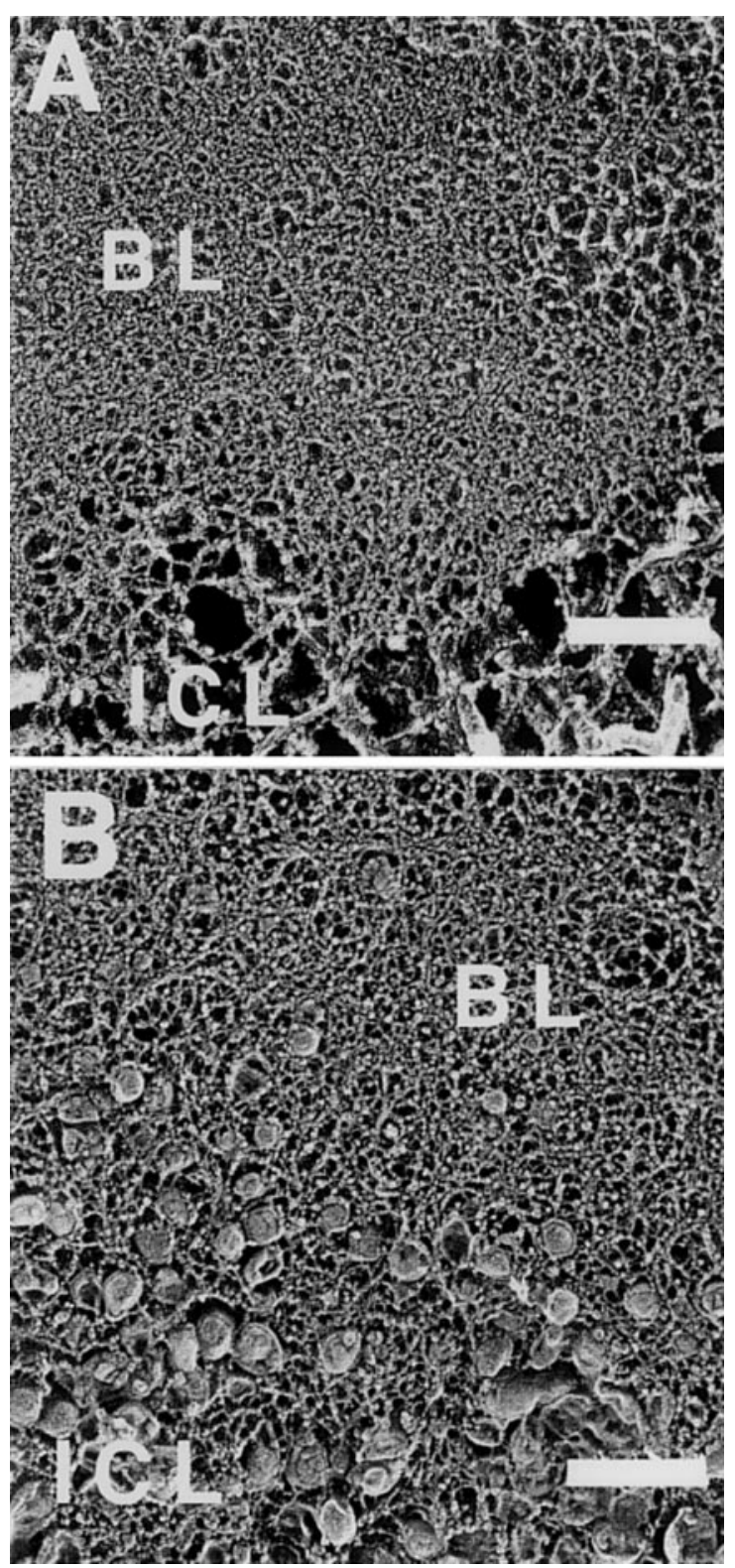

FigURE 7. QFDE oblique sections through basal lamina (BL) of the RPE from a (A) 27-year-old and (B) a 78-year-old eye. In the older eye, there appeared to be more open space in the basal lamina and basallamina-like filaments extended to and surround lipid rich particles embedded in the matrix. Scale bar, $300 \mathrm{~nm}$. 


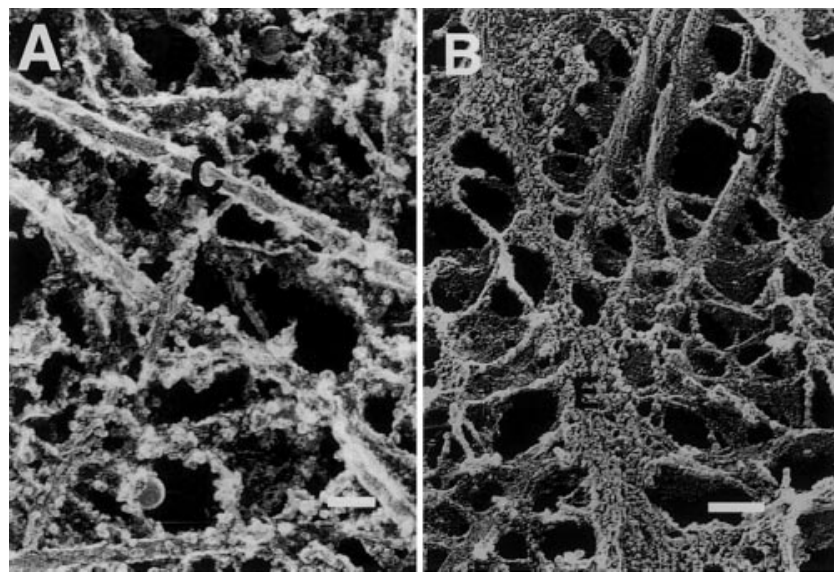

Figure 8. ICLs of a 41-year-old (A) and a 78-year-old (B) eye imaged by QFDE. Note the fine-scale decoration of collagen (C) components of the ECM in the young eye. In the older eye, these moieties were reduced or absent. E, elastin-like material. Scale bar, $100 \mathrm{~nm}$.

tion of lipoproteins. ${ }^{33,34}$ However, those particles were rich in unesterified cholesterol and contained little esterified cholesterol, ${ }^{35-38}$ which is abundant in Bruch's membrane lipid particles. In our study, the shell on the particle's exterior may represent a small component of unesterified cholesterol solubilized in phospholipid. ${ }^{26,39}$ Alternatively, this morphology could signify surface and core components typical of lipoprotein particles. ${ }^{40,41}$ The significance of lipid particle morphology revealed by QFDE will become clearer when these particles are isolated and characterized.

The source of the cholesterol-containing particles found in Bruch's membrane is unknown. A high proportion of esterified cholesterol in relation to total cholesterol is consistent with direct infusion of plasma low-density lipoproteins (LDLs) into Bruch's membrane, a process that occurs in other connective tissues. ${ }^{2}$ However, preliminary analysis indicates that the distribution of cholesteryl esters in Bruch's membrane does not resemble that of plasma LDL. ${ }^{42}$ Further, human RPE has potential for biosynthesis of apolipoprotein B (apoB), the principal protein of very-low-density lipoprotein (and its metabolic product, LDL) and intestinal chylomicrons, and apoB immunoreactivity can be found in normal Bruch's membrane. ${ }^{43}$ These data raise the possibility that the RPE assembles large apoB-containing lipoproteins for basal release into Bruch's membrane. Definitive identification of the source of esterified cholesterol in Bruch's membrane clearly requires further study.

Nevertheless, the details of where these lipid rich particles are found in relation to the RPE and its basal lamina, as shown in our study, are important for evaluating possible mechanisms by which cholesterol arrives in Bruch's membrane. The size of the lipid-containing particles (roughly 80-100 nm) is much greater than the typical interfibrillar spacing in the basal lamina (Figs. 2B, 2D, 3B, 3D, 7), and few particles were detected within the basal lamina. Assuming the basal lamina to be continuous and to be incapable of deforming sufficiently to allow the passage of these large particles, this suggests that if these particles originate in the RPE, they are unlikely to have passed through the basal lamina in this form. One possibility is that very small lipid particles pass through the basal lamina and coalesce to form the large particles that constitute the lipid wall. Another possibility is that the particles deform as they squeeze through the basal lamina of the RPE. Chylomicrons, lipoprotein particles more than $75 \mathrm{~nm}$ in diameter formed by intestinal enterocytes, are known to pass through a basal lamina en route to lymphatic channels. ${ }^{44} \mathrm{~A}$ third possibility is that these particles are derived from the choriocapillaris and do not pass through the RPE basal lamina, although, as mentioned, recent biochemical analysis has cast doubt on the hypothesis that these particles are exclusively blood derived. ${ }^{42,43}$

Although quantitative data are needed from more eyes to confirm our observations, our findings allow us to evaluate the potential contribution of age-related differences in extracellular matrix components to the observed age-related changes in hydraulic conductivity of Bruch's membrane. Previous examination of the age-related decrease in hydraulic conductivity of Bruch's membrane has emphasized fibrillar collagen in the ICL of Bruch's membrane. ${ }^{22}$ However, preliminary hydrodynamic analyses indicate that the collagen fibrils of this layer would present negligible hydrodynamic flow resistance. ${ }^{45}$ In the present study, we saw that labeling of individual fibers in this region was decreased in the older eyes (Fig. 8), a finding that is not consistent with an age-related decrease in hydraulic conductivity. Our QFDE images (Figs. 2B, 2D, 3B, 2D, 4, 6) indicate that the weave of the RPE basal lamina and staining of collagen fibrils in this region should be considered contributors to resistance as well, because the interfibrillar spaces are much smaller than those seen in the ICL. However, the RPE basal lamina does not appear any more tightly woven in older adults, and it is possibly even less tight than it is in the eyes of the younger adults we examined (Figs. 6, 7). Thus, this finding is also inconsistent with an age-related decrease in hydraulic conductivity.

It is logical to hypothesize that transport of water and hydrophilic moieties across Bruch's membrane in the older eyes examined in this study would be compromised by the lipid wall. The relationship of lipid accumulation in Bruch's membrane to the formation of basal linear deposits and drusen and the progression to neovascularization remains to be determined. However, the degeneration and death of RPE and photoreceptors in the overlying retina are aspects of ARM pathobiology that could be consequences of the development of the physical barrier described in this study. ${ }^{23}$ The precise physicochemical circumstances that allow lipid nucleation and aggregation confined to this narrow layer in the extracellular matrix must be investigated to elucidate how a lipid barrier could contribute to the pathogenesis of late ARM. A detailed engineering analysis of tissue transport properties based on high quality morphologic information afforded by QFDE has recently been achieved for human cornea. ${ }^{27}$ Our current results indicate that a similar approach to understanding transport across Bruch's membrane is now warranted.

\section{References}

1. Council NAE. Vision Research-A National Plan: 1999-2003, Executive Summary. Bethesda, MD: National Institutes of Health; $1998 ; 18$.

2. Curcio CA, Millican CL, Bailey T, Kruth HS. Accumulation of cholesterol with age in human Bruch's membrane. Invest Opbthalmol Vis Sci. 2001;42:265-274.

3. Haimovici R, Gantz DL, Rumelt S, Freddo TF, Small DM. The lipid composition of drusen, Bruch's membrane, and sclera by hot stage polarizing light microscopy. Invest Ophthalmol Vis Sci. 2001;42: 1592-1599.

4. Karwatowski W, Jeffried T, Duance V, Albon J, Bailey A, Easty D. Preparation of Bruch's membrane and analysis of the age-related changes in the structural collagens. Br J Ophthalmol. 1995;79: $944-952$.

5. Handa JT, Verzijl N, Matsunaga H, et al. Increase in the advanced glycation end product pentosidine in Bruch's membrane with age. Invest Ophthalmol Vis Sci. 1999;40:775-779.

6. Guo L, Hussain AA, Limb GA, Marshall J. Age-dependent variation in metalloproteinase activity of isolated human Bruch's membrane and choroid. Invest Ophthalmol Vis Sci. 1999;40:2676-2682. 
7. Pauleikhoff D, Harper CA, Marshall J, Bird AC. Aging changes in Bruch's membrane: a histochemical and morphological study. Ophthalmology. 1990;97:171-178.

8. Holz FG, Sheraidah G, Pauleikhoff D, Bird AC. Analysis of lipid deposits extracted from human macular and peripheral Bruch's membrane. Arch Ophthalmol. 1994;112:402-406.

9. Sarks SH. Ageing and degeneration in the macular region: a clinicopathological study. Br J Ophthalmol. 1976;60:324-341.

10. Curcio CA, Millican CL. Basal linear deposit and large drusen are specific for early age-related maculopathy. Arch Ophthalmol. 1999;117:329-339.

11. Hogan MJ, Alvarado JA, Weddell JE. Histology of the human eye An Atlas and Textbook. Philadelphia: WB Saunders; 1971;328363.

12. Feeney-Burns L, Ellersieck MR. Age-related changes in the ultrastructure of Bruch's membrane. Am J Ophthalmol. 1985;100:686697.

13. Killingsworth MC. Age-related components of Bruch's membrane. Graefes Arch Clin Exp Ophthalmol. 1987;225:406-412.

14. van der Schaft TL, Mooy CM, de Bruijn WC, Oron FG, Mulder PGH, de Jong PTVM. Histologic features of the early stages of age-related macular degeneration. Ophthalmology. 1992;99:278-286.

15. Ramrattan RS, van der Schaft TL, Mooy CM, de Bruijn WC, Mulder PGH, de Jong PTVM. Morphometric analysis of Bruch's membrane, the choriocapillaris, and the choroid in aging. Invest Ophthalmol Vis Sci. 1994;35:2857-2864.

16. Smith E. Relationship between plasma lipids and arterial tissue lipids. Nutr Metab. 1973;15:17-26.

17. Katz SS, Small, DM. Isolation and partial characterization of the lipid phases of human atherosclerotic plaques. J Biol Chem. 1980; 255:9753-9759.

18. Guyton JR, Bocan TMA, Schifani TA. Quantitative ultrastructural analysis of perifibrous lipid and its association with elastin in nonatherosclerotic human aorta. Arteriosclerosis. 1985;5:644652.

19. Gaynor PM, Zhang WY, Salehizadeh B, Pettiford B, Kruth HS Cholesterol accumulation in human cornea: evidence that extracellular cholesteryl ester-rich lipid particles deposit independently of foam cells. J Lipid Res. 1996;37:1849-1861.

20. Kruth H. Lipoprotein cholesterol and atherosclerosis. Curr Mol Med. 2001;6:633-653.

21. Bird AC, Marshall, J. Retinal pigment epithelial detachments in the elderly. Trans Ophthalmol Soc UK. 1986;105:674-682.

22. Marshall J, Hussain AA, Starita C, Moore DJ, Patmore AL. Aging and Bruch's membrane. In: Marmor MF, Wolfensberger TJ, eds. The Retinal Pigment Epithelium: Function and Disease. New York: Oxford University Press; 1998;669-692.

23. Jackson GR, Owsley C, Curcio CA. Photoreceptor degeneration and dysfunction in aging and age-related maculopathy. Ageing Res Rev. 2002;1:381-396.

24. Moore DJ, Hussain AA, Marshall J. Age-related variation in the hydraulic conductivity of Bruch's membrane. Invest Ophthalmol Vis Sci. 1995;36:1290-1297.

25. Starita C, Hussain A, Pagliarini S, Marshall J. Hydrodynamics of ageing Bruch's membrane: implications for macular disease. $\operatorname{Exp}$ Eye Res. 1996;62:565-572.

26. Guyton JR, Klemp KF. Ultrastructural discrimination of lipid droplets and vesicles in atherosclerosis: value of osmium-thiocarbohydrazide-osmium and tannic acid-paraphenylenediamine techniques. J Histochem Cytochem. 1988;36:1319-1328.

27. Overby D, Ruberti J, Gong H, Freddo T, Johnson M. Specific hydraulic conductivity of corneal stroma as seen by quick-freeze/ deep-etch. J Biomech Eng. 2001;123:154-161.
28. Menco BP. Freeze-fracture, deep-etch, and freeze-substitution studies of olfactory epithelia, with special emphasis on immunocytochemical variables. Microsc Res Tech. 1995;32:337-356.

29. Phillips T, Boyne A. Liquid nitrogen-based quick freezing: experiences with bounce-free delivery cholinergic nerve terminals to a metal surface. J Electron Microsc Technique. 1984;1:9-29.

30. Ou B, Ohno S, Yamabayashi S, Chen H, Fujii Y, Tsukahara S. Ultrastructural study of basal lamina of retinal pigment epithelium. Ophthalmic Res. 1995;27:158-162.

31. Call TW, Hollyfield JG. Sulfated proteoglycans in Bruch's membrane of the human eye: localization and characterization using cupromeronic blue. Exp Eye Res. 1990;51:451- 462.

32. Nakaisumi Y, Hogan M, Feeney L. The ultrastructure of Bruch's membrane. III. The macular area of the human eye. Arch Ophthalmol. 1964;72:395-400.

33. Frank JS, Fogelman AM. Ultrastructure of the intima in WHHL and cholesterol-fed rabbit aorta prepared by ultra-rapid freezing and freeze-etching. J Lipid Res. 1989;30:967-978.

34. Nievelstein PFEM, Fogelman AM, Mottino G, Frank JS. Lipid accumulation in rabbit aortic intima 2 hours after bolus infusion of low density lipoprotein: a deep etch and immunolocalization study of ultrarapid frozen tissue. Arteriosclerosis Thrombosis. 1991;11: 1795-1805.

35. Kruth HS. Subendothelial accumulation of unesterified cholesterol: an early event in atherosclerotic lesion development. Atherosclerosis. 1985;57:337-341.

36. Simionescu N, Vasile E, Lupu F, Popescu G, Simionescu M. Prelesional events in atherogenesis: accumulation of extracellular cholesterol-rich liposomes in the arterial intima and cardiac valves of the hyperlipidemic rabbit. Am J Patbol. 1986;123:109-125.

37. Chao FF, Blanchette-Mackie EJ, Skarlatos SI, et al. Unesterified cholesterol-rich lipid particles in atherosclerotic lesions of human and rabbit aortas. Am J Patbol. 1988;131:73-83.

38. Schwenke D. Selective increase in cholesterol at atherosclerosissusceptible aortic sites after short-term cholesterol feeding. Arteriosclerosis Thrombosis Vasc Biol. 1995;15:1928-1937.

39. Kruth H. Cholesterol deposition in atherosclerotic lesions. In: Bittman R, ed. Cholesterol: Its Functions and Metabolism in Biology and Medicine. New York: Plenum; 1997:319-362. Subcellular Biochemistry; Vol. 28.

40. Havel RJ, Kane JP. Introduction: structure and metabolism of plasma lipoproteins. In: Scriver CR, Beaudet AL, Sly WS, Valle D, eds. The Metabolic and Molecular Basis of Inherited Disease. New York: McGraw-Hill; 2001:2707-2716.

41. Segrest JP, Jones MK, De Loof H, Dashti N. Structure of apolipoprotein B-100 in low density lipoproteins. J Lipid Res. 2001;42:13461367.

42. Curcio C, Bradley K, Guidry C, et al. A local source for esterified cholesterol (EC) in human Bruch's membrane (BrM). ARVO Abstract 862, 2002.

43. Malek G, Li C-M, Guidry C, Medeiros N, Curcio C. Apolipoprotein $\mathrm{B}$ in cholesterol-containing drusen and basal deposits in eyes with age-related maculopathy. Am J Pathol. 2003;162:413- 425.

44. Cardell RR Jr. Badenhausen S, Porter KR. Intestinal triglyceride absorption in the rat: an electron microscopical study. J Cell Biol. 1967;34:123-155.

45. Ruberti JW, Curcio CA, Menco B, Johnson M. Quick freeze/deep etch (QFDE): a better imaging method to extract micro-scale specific hydraulic conductivity (SHC) from Bruch's membrane [ARVO Abstract]. Invest Ophthalmol Vis Sci. 2001;42:S224. Abstract nr 1204. 